\title{
EL ESPACIO EN LA PERSPECTIVA DE UN NUEVO ORDEN POLITICO-ESTRATEGICO INTERNACIONAL
}

\author{
Agustín Toro Dávila
}

\begin{abstract}
Frente a un posible nuevo orden internacional, es importante establecer la influencia del espacio ultraterrestre en éste. En el esquema de la Guerra Fría, las relaciones entre los Estados se caracterizan por un rígido bipolarismo político-estratégico, en el cual ambos bloques tratan de sobrepasarse mutuamente para romper el equilibrio de poder. Si en algunos campos había paridad, ésta no existía en el espacio aéreo y ultraterrestre, desatándose una carrera entre las dos superpotencias. En este sentido, el autor plantea que ante un eventual nuevo ordenamiento político del mundo, el uso militar del espacio alcanza especial trascendencia. Actualmente, Estados Unidos es la potencia que domina el espacio. La Unión Soviética desintegrada y los países en desarrollo se encuentran marginados, lo que en su opinión podría producir un apartheid científico-tecnológico que los dejaría en una desventajosa posición.
\end{abstract}

\section{Introducción.}

El enunciado del tema nos plantea dos interrogantes a las que es preciso responder antes de establecer la relación entre los dos elementos básicos de este estudio: las características y posibilidades de existencia de un orden internacional diferente al que vivió el mundo por 45 años y el uso del espacio ultraterrestre como factor influenciador de ese nuevo orden.

El orden internacional es el producto de una política mundial y sobre ésta así como sobre las políticas y las relaciones internacionales existen muchas y muy variadas definiciones, sin embargo se produce un consenso mayoritario en la determinación de los objetivos que animan a la política exterior de todo Estado. Según los autores Pablo Valdés y Juan Salazar estos objetivos serían fundamentalmente los siguientes: ${ }^{1}$

\footnotetext{
${ }^{1}$ Pablo Valdés y Juan Salazar, Polftica mumdial contemporánea, (Santiago: Editorial Andrés Bello, 1979).
} 
i) preservación de su integridad territorial (espacios terrestre, marítimo y aéreo);

ii) mantenimiento de su independencia política; y

iii) consecución de sus intereses nacionales.

Ya que tanto la soberanía (integridad territorial) como la seguridad (independencia política) se constituyen en un interés primario para cualquier Estado, resulta que son precisamente los "intereses nacionales", incluidos entre ellos a los anteriormente mencionados, los que mejor se identifican con los objetivos de una política exterior.

Para Hans Morgenthau, ${ }^{2}$ mientras la comunidad internacional esté políticámente organizada en naciones, el interés nacional será, sin duda, la última palabra en la política mundial. Las naciones deben

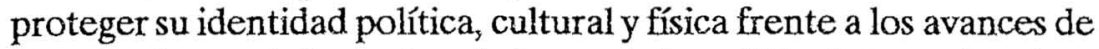
otras naciones, de lo cual se deduce que la política internacional no es más que la interacción de los intereses nacionales, muchas veces contrapuestos, entre diferentes Estados.

Las relaciones entre las naciones en el plano internacional tienen la característica de ser extraordinariamente cambiantes ya que vinculan a los Estados conformando una realidad que está en continua transformación, de acuerdo con las prioridades que se presentan en los intereses que cada uno de ellos desee alcanzar en un presente determinado y hacia un futuro presupuestado. Sin embargo, en ellos se revelan ciertas constantes ya que estas relaciones internacionales no son fenómenos aislados sino que se desarrollan dentro de un esquema y un sistema a los cuales están sujetas. ${ }^{3}$

El esquema, según Valdés y Salazar ${ }^{4}$ "corresponde a las estructuras de las relaciones internacionales formadas por los diferentes hechos que caracterizan la política internacional en una época determinada". Por su parte, Stanley Hoffman ${ }^{5}$ sostiene que "un sistema internacional es un modelo (pauta) de relaciones entre las unidades básicas de la política mundial, que se caracteriza por el alcance de los objetivos perseguidos por dichas unidades y las empresas cumplidas entre ellas así como por los medios empleados a fin de llevar adelante dichos objetivos y tareas. El modelo (pauta) está determinado en muy buena parte por la estructura del mundo, la naturaleza de las fuerzas

\footnotetext{
.2 Hans Morgenthau, Polftica entre las Naciones, (Buenos Aires: Grupo Editorial Latinoamericano).

${ }^{3}$ Pablo Valdes y Yuan Salazar, op. cit

${ }^{4}$ Bbid.

$s_{\text {Ibid }}$
} 
en juego dentro y fuera de las unidades mayores, las posibilidades nacionales y las normas de distribución del poder".

Conforme a estos conceptos es posible definir que la política internacional es el conjunto de acciones y situaciones que se generan entre actores internacionales (Estados, coaliciones, organismos internacionales, grupos de presión, etc.), los que constituyen elementos de determinados esquemas de relaciones exteriores y cuyo comportamiento se ordena en función de variables de un sistema $\rightarrow$ un subsistema-internacional" 6

El término de la Segunda Guerra Mundial produjo cambios notables tanto en el esquema como en el sistema internacionales que operaron desde la finalización de la Primera Gran Guerra, así como también los actores modificaron sus posiciones de acuerdo a los poderes nacionales que algunos alcanzaron y que otros perdieron o redujeron. Las potencias europeas que habían sido actores de primer rango se debilitaron; Japón, Alemania e Italia que habían quebrantado el equilibrio en su beneficio en procura de concretar un revisionismo del statu quo existente, terminaron la guerra destruidos poítica, económica y militarmente y surgieron dos actores como superpotencias de primer rango, Estados Unidos y la Unión Soviética.

El enfrentamiento de los intereses de estas dos superpotencias, una, Estados Unidos buscando la mantención del statu quo, la otra, la Unión Soviética llevando adelante una política de revisionismo o de revolución, configuraron por 45 años, un esquema de relaciones internacionales que fue la Guerra Fría y un sistema internacional de un rígido bipolarịsmo político-estratégico.

En relación con el espacio, se puede precisar que no es un terreno exclusivamente militarizado en el sentido convencional de la palabra ${ }^{7}$ por cuanto no existen armas que giren en torno a la tierra o fuerzas militares permanentemente estacionadas en el espacio. Los satélites que se lanzaban regularmente al espacio por parte de las dos superpotencias y que perseguían fines militares aparecían de carácter benigno e inofensivo; no poseían los medios para atacar a otros objetos en el espacio y más bien promovían la paz y la seguridad internacionales al suministrar a las superpotencias informaciones precisas sobre actividades militares recíprocas así como la alerta de

\footnotetext{
${ }^{6}$ Ibid)

${ }^{7}$ Paul B. Stares, "Tecnología Espacial. Avances relacionados con al seguridad"; Revista Jurfdica de Las Naciones Unidas.
} 
un ataque y conductas seguras para la gestión de las crisis; de esta manera, ellos apoyaban la disuasión, la verificación del control de los armamentos y la estabilidad estratégica en general.

\section{La carrera espacial.}

Ya durante las conversaciones de paz al término de la Segunda Guerra, se inició el proceso de ruptura entre las potencias vencedoras. Estados Unidos había desarrollado el mayor esfuerzo bélico con operaciones conjuntas con sus aliados en Europa, el Reino Unido, Francia y la Unión Soviética, y prácticamente propias en la confrontación con el Japón; era la mayor potencia militar y económica y tenía la exclusividad de la capacidad nuclear. Estas circunsitancias llevaban a pensar que en Estados Unidos debería haberse gestado la resolución de la paz. Sin embargo, fue la otra superpotencia, Unión Soviética, la que con una mayor agresividad diplomática logró imponer sus antiguas pretensiones de conquistar la hegemonía en gran parte de Europa y obtener beneficios políticos y territoriales en el Lejano Oriente. Esta contraposición de objetivos generó una ruptura que dio origen a la formación de dos bloques o mundos antagónicos cuyas acciones y reacciones mantuvieron a la humanidad en la incertidumbre y en un alto grado de inseguridad internacional por cerca de medio siglo.

Cada una de estas superpotencias, líderes de los mundos occidental y oriental, llevó adelante una política de preparación de sus potenciales militares, tratando una de sobrepasar a la otra en procura de romper el equilibrio de poder y así imponer su voluntad: o se mantenía el statu quo o triunfaba el revisionismo y la revolución. En los campos militares terrestres y navales se mantenía una paridad, pero quedaba el espacio aéreo y ultraterrestre para lograr allí una ventaja.

La utilización de satélites artificiales en el ámbito militar por parte de los países potencia, es una circunstancia que si bien tuvo orígenes anteriores, se materializó en octubre de 1957 con la colocación en órbita del primer satélite artificial de la tierra, el Sputnik I por parte de la Unión Soviética, la que así demostró al mundo y especialmente a su gran rival, Estados Unidos, su mayor avance en capacidad científica y tecnológica. Con esto, el espacio ultraterrestre 
se incorporó definitivamente como un elemento más a considerar en la definición de los poderes nacionales.

Como réplica al Sputnik I, en abril de 1959, Estados Unidos puso en órbita el satélite Discover 2 con capacidad para la toma de fotografías de objetos en la tierra.

De esta manera, según el profesor Ulises Faúndez se inició la denominada "carrera espacial entre las dos superpotencias, la cualles ayudó básicamente en dos aspectos globales: provocó una espiral ascendente en el desarrollo científico-tecnológico y les sirvió de excelente instrumento de prestigio político internacional" ${ }^{8}$ Pocos meses después, en abril de 1959, la Unión Soviética envió un satélite no tripulado hacia la luna y en Estados Unidos, la administración del Presidente Kennedy, comenzó a impulsar un proyecto para lograr la presencia de un norteamericano en ese mismo planeta.

En la década de los años sesenta, tanto Estados Unidos como la Unión Soviética lanzan al espacio ultraterrestre satélites tripulados por seres humanos, sin embargo con todos ellos no se logra proyectar soluciones en beneficio de la humanidad. Sobre este particular, el mismo profesor Faúndez ya citado, ${ }^{9}$ establece que se percibe una diferenciación de objetivos en los proyectos realizados en la década del sesenta con la siguiente, la del setenta; en la primera se proyectaban fines aplicados hacia el universo, en la segunda los fines se aplicaron hacia la tierra, iniciándose la era del "satélite artificial circunsterrestre" con capacidad para observar y captar diversas situaciones y fenómenos que ocurrían en nuestro planeta, y no solamente con miras hacia una utilización exclusiva en el campo militar sino con proyecciones hacia las áreas económicas de los sectores privados. Los beneficios resultaban así más concretos y era posible compensar los altos costos con recursos obtenidos de la venta de datos y de usos.

La competencia de las dos superpotencias continuó durante toda la década de los años ochenta; en lo militar se había creado un sistema de inteligencia que proporcionaba antecedentes sobre actividades militares recíprocas. Así, los satélites artificiales fueron siendo cada vez más útiles como elementos auxiliares de guerra para los altos comandos de las Fuerzas Armadas.

Estos podian ser empleados en todo tipo de conflictos convencionales localizados y hasta en la probabilidad de una guerra nucleạr.

\footnotetext{
${ }^{8}$ Ulises Faúnđez, "Tecnología espacial y defensa", Revisua Sociedad y Fuerzas Armadas.

${ }^{9} \mathrm{Ibid}$
} 
Los satélites de reconocimiento se utilizaban y se están utilizando para detectar, seguir la trayectoria y localizar a fuerzas militares terrestres, navales y bases aéreas; los satélites đe navegación pueden guiar de manera activa incluso a las municiones "más estúpidas" hacia sus objetivos con una gran precisión. El aumento de las aplicaciones militares de los sistemas espaciales ha incrementado, lógicamente, los incentivos para negar sus beneficios al enemigo en tiempo de guerra y también se ha producido la necesidad de proteger a los satélites propios en contra de posibles ataques. ${ }^{10}$

Tanto la Unión Soviética como Estados Unidos crearon sistemas de armas antisatélites lo cual significaba que los satélites de ambas superpotencias podrían ser objeto de ataque por parte del adversario. La propuesta del Presidente Ronald Reagan, la Iniciativa de Defensa Estratégica o "Guerra de las Galaxias", dada a conocer en marzo de 1983, abrió nuevas posibilidades para los sistemas de defensa contra misiles balísticos.

La actividad espacial, en todos los campos civiles y en los militares ha continuado realizándose, prácticamente en forma exciusiva, por parte de las superpotencias. Sin embargo el quebrantamiento de la Unión Soviética y su desintegración afectó en gran medida su capacidad económica para apoyar acciones en el espacio y es posible que no pueda reanudarlas independientemente por más tiempo.

El espacio ultraterrestre en su aspecto militar podría aparecer como un elemento propio o exclusivo de Estados Unidos, sin embargo, el surgimiento de potencias económicas, como es el caso de Japón y los países de Europa Occidental, pueden romper esa exclusividad.

\section{El uso militar de los satélites.}

El empleo militar del èspacio es hoy una realidad que se hace cada día más efectiva con el vertiginoso avance de la ciencia y la tecnología; este empleo tiene su campo tanto en períodos de paz como durante eventuales procesos de crisis bélica y es por ello que alcanza especial trascendencia en un posible nuevo ordenamiento político del mundo.

Como ya se expresó anteriormente, el mundo bipolar que vivimos hasta el comienzo de esta década de los años noventa dejó de

\footnotetext{
${ }^{10}$ Paul B. Stares, op. cit.
} 
A. Toro D. / El espacio en la perspectiva de un nuevo ...

existir potencialmente cuando la Unión Soviética sufrió el quebrantamiento propio de todo Estado dentro del cual se desintegran sus factores básicos. El espacio era precisamente uno de los campos de rivalidad entre ella y Estados Unidos y, tal vez, fue precisamente la gran exigencia económica de la carrera espacial, una de las muchas causas que llevó a Moscú al colapso.

Para Paul B. Stares, ${ }^{11}$ los satélites militares cumplen funciones tanto en tiempo de paz como de guerra. En relación con las actividades de tiempo de paz expresó que: "Son cruciales para la reunión de información de las superpotencias y para las actividades de vigilancia de la limitación de los armamentos, coadyudan en la gestión de las crisis internacionales, facilitan la planificación y sirven para dar la alerta de un ataque".

Las actividades y funciones que cumplen los satélites militares en tiempo de paz se pueden clasificar en:12

- Reunión de información y supervisión de limitación de los armamentos. Se emplean satélites de reconocimiento con una fuente segura de información por su capacidad fotográfica, ya que pueden captar con gran exactitud imágenes de determinados sectores del planeta que resultan totalmente ocultos a cualquier otro sistema de reconocimiento.

Las dos superpotencias se vigilaban mutuamente sus diferentes sistemas de armamentos y emplazamientos, fuerzas terrestres, navales y aéreas, y ubicaciones y capacidades.

- Planificación de la guerra. Toda planificación de guerra requiere de la mantención de antecedentes del potencial militar del adversario exactos y actualizados. Lo primero, para determinar las capacidades de posibles acciones o reacciones del adversario e incluso obtener datos sobre sus eventuales operaciones ofensivas o defensivas de acuerdo a las dispositivos señalizados; lo segundo, por cuanto las fuerzas militares no son estáticas y se desplazan permanentemente para evitar una acción contraria sorpresiva. Las informaciones sobre emplazamientos de misiles estratégicos intercontinentales presentan un cuadro preciso sobre la intencionalidad de ellos en cuanto se refiere a los presuntos objetivos sobre los cuales pueden emplearse. Asimismo la ubicación de radares, especialmente los móviles, dan orientaciones para adop-

${ }^{11}$ Ibid.

${ }^{12}$ Ibid. 
tar con tiempo contramedidas electrónicas de interferencia y técnicas de desorientación.

- Gestión de crisis. Tanto Estados Unidos como la ex-Unión Soviética tenían zonas de influencia en áreas territoriales y marítimas del Tercer Mundo que les permitían un extenso despliegue de sus fuerzas militares. En estas áreas podían producirse acontecimientos o conflictos que, por estar influenciados o apoyados por alguno de ellos, podían amenazar la estabilidad de la paz mundial. Los sistemas de satélites de comunicaciones servían a los gobiernos de las superpotencias para mantener un conocimiento anticipado de los posibles conflictos y establecer contactos directos con sus fuerzas militares ubicadas en lejanos y poco accesibles lugares del planeta, de tal manera de moverlas con rapidez y precisión hacia los lugares en los cuales podría ser decisiva su participación para evitar los conflictos o apoyarlos según fuera necesario o conveniente a los intereses político-estratégicos.

- Alerta anticipada. En el período de Guerra Fría cuando las superpotencias mantenían entre ellas un recelo permanente, la posibilidad de recibir una alerta anticipada de las intenciones ofensivas del adversario significaba la posibilidad de tener tiempo suficiente para adoptar las medidas pertinentes para reaccionar en la forma más efectiva.

Las variaciones de los emplazamientos normales de fuerzas nucleares, los movimientos de las fuerzas navales, aéreas y terrestres, los cambios de las estructuras de comunicaciones eran indicaciones válidas para determinar la intención ofensiva del enemigo. Asimismo, los satélites de alarma anticipada podrían informar del lanzamiento de misiles nucleares intercontinentales con una antelación aproximada de 25 minutos antes de la llegada de ellos a sus objetivos. Esta antelación sólo podía ser aproximada ya que sería mucho menor, no más de 15 minutos, en caso de operarse misiles de alcance intermedio desde submarinos.

- Previsiones metereológicas. Son especialmente válidas para las operaciones militares aéreas y navales en tiempo de guerra, por cuanto proporcionan antecedentes bastante precisos para la seguridad de la navegación tanto aérea como marítima.

En cuanto a un posible uso de satélites en tiempo de guerra, esto se estima como indispensable para el correcto y oportuno empleo de las fuerzas militares, navales, aéreas y terrestres. 
Los antecedentes sobre los movimientos adversarios, presuntos objetivos, las operaciones estratégicas y acciones tácticas en ejecución, permiten a los comandos tomar sus resoluciones con un alto grado de seguridad.

Es verdad que directamente entre ambas superpotencias no hubo un enfrentamiento, pero sí se produjeron conflictos localizados en los que los satélites jugaron un papel decisivo para los intereses que en ellos tenían la ex-Unión Soviética o Estados Unidos.

Posiblemente, una constante situación de equilibrio de los potenciales de ambas superpotencias en cuanto a sistemas de satélites militares significó un importante aporte para mantener la disuasión. En una guerra entre ellas con empleo de armas nucleares, la sorpresa era el factor de la victoria; el conflicto podría resolverse en muy breve tiempo si una lograba vulnerar la seguridad de la otra asestando un primero y decisivo golpe a su infraestructura militar y económica. Al dificultarse lograr esa sorpresa, las opciones de impunidad inicial quedaban anuladas o disminuidas considerablemente y debía esperarse una rápida reacción con la cual se buscara compensar el desequilibrio iniciai; la represalia significaba recibir los mismos efectos poco tiempo después que los hubiera recibido el atacádo.

La importancia que fueron adquiriendo los sistemas espaciales en la planificación y las posibles operaciones bélicas, generó un interés creciente en ambas superpotencias para impedir el uso de ellas por parte del adversario. De aquí nacieron las armas espaciales.

Para el autor Paul B. Stares ya citado, existen tres procedimientos especialmente importantes para negar al adversario los beneficios de su sistema de satélites:

- Ataque al satélite adversario: resulta ser la forma o procedimiento más directo, pero podría significar la iniciación de un conflicto que podría haberse evitado. La destrucción de un satélite durante el desarrollo de un conflicto convencional entre las superpotencias podría ser considerado como la iniciación de un ataque nuclear. Por otra parte es necesario considerar que un satélite destruido puede ser reemplazado por otro ya en órbita o por uno preparado para un lanzamiento rápido.

- Interrupción de las comunicaciones con la nave espacial por medios de interferencias electrónicas. Esta forma es menos directa que la anterior y no podría garantizar el buen éxito ante ataques más directos. Combinando este procedimiento con el engaño 
(proporcionar informaciones falsas el satélite), se podrían obtener mejores resultados en casos aislados.

- Ataques a las estaciones terrestres que controlan a los satélites y reciben los datos que ellos proporcionan. Sería un procedimiento eficaz en una guerra convencional de mayor duración en tiempo, pues estos ataques deben prepararse minuciosamente y ser realizados por fuerzas especiales todo lo cual demanda una planificación minuciosa y un proceso relativamente largo de ejecutar.

El vertiginoso desarrollo de la ciencia y de la tecnología espaciales permitió la creación de sistemas de defensa en contra de misiles balísticos. Los misiles intercontinentales y los lanzados desde submarinos se desplazan por el espacio ultraterrestre llegando a alcanzar más de $1.000 \mathrm{kms}$. de altura en la curvatura máxima de sus trayectorias. Entre los sistemas de defensa contra estos misilès pueden considerarse los basados en el espacio para detectar, seguir y destruir misiles en las primeras fases de vuelo y sistemas basados en tierra para verificar sus trayectorias y destruirlos en sus fases terminales.

Dentro del campo científico y tecnológico se continúa estudiando sistemas de defensa estratégica y ya se ha llegado a establecer diferentes alternativas posibles de materializar, aun cuando se enfrentan enormes dificultades por los altos costos sin tener una seguridad absoluta de buen éxito

La mayor eficacia que adquieren los satélites artificiales en apoyo del aumento del poder combativo de las fuerzas militares es un incentivo para la creación de armas antisatélites, como también la mayor fuerza destructiva de los misiles balísticos y sus largos alcances significan la necesidad de crear sistemas de defensa en contra de ellas. Harold Brown, ex-Secretario de Defensa de Estados Unidos se refirió a la Iniciativa de Defensa Estratégica de su país con una frase que define exactamente el problema que se vive entre la acción y la reacción: lo que es deseable no es factible y lo que es factible no es necesariamente favorable.

\section{El espacio y el nuevo orden internacional.}

Después de la victoria obtenida por las fuerzas aliadas lideradas por Estados Unidos en la Guerra del Golfo, emergió, especialmente en Estados Unidos, la idea del establecimiento de un nuevo orden internacional. Sin embargo en la reunión cumbre de Londres reali- 
zada en julio de 1991, los siete países más industrializados del mundo firmaron una declaración conjunta en la cual no se acepta el concepto de un nuevo orden sino que el fortalecimiento del existente.

¿Se trata efectivamente de un nuevo orden internacional y cuáles serían sus elementos y actores fundamentales? ¿Qué rol jugará el Consejo de Seguridad de las Naciones Unidas y cuál será la libertad de acción que éste tendrá para adoptar sus propias resoluciones y materializarlas? ¿Será el dominio político-estratégico del espacio ultraterrestre un apartheid científico-tecnológico impulsado por la única superpotencia y en menor medida por potencias del mundo industrializado? ¿Cómo afectará este apartheid la soberanía de las naciones del mundo no industrializado y sin capacidad económica para desarrollar sus capacidades científico-tecnológicas? De estas interrogantes muy pocas son las que pueden tener una respuesta precisa y segura, puesto que la realidad es que las diferentes visiones que se tienen sobre el nuevo orden internacional y el vertiginoso avance en la carrera por el espacio y sus cambiantes alternativas, sólo permite analizar y discutir posibilidades más que determinar verdades absolutas.

Robert Gates, Vice-Consejero de Seguridad Nacional del Presidente Bush (recientemente designado y Director de la CIA), se refiere al nuevo orden internacional bajo el liderazgo norteamericano: "Hoy en día nadie pone en discusión la realidad de una superpotencia. En todos las medidas comunes de poder nacional, económica, militar, cultural, política e incluso filosófica no tenemos quien pueda retarnos". 13

Sobre el rol de las Naciones Unidas, el mismo Gates se refirió al papel que ella jugó en la Guerra del Golfo en el sentido de que, por primera vez, desempeñó la función de "dirigir y sancionar una resistencia colectiva a un agresor" y que "los pilares de una nueva era internacional son las posibilidades de continuar desempeñando esta función y la nueva disposición de muchas naciones de contribuir con dinero y tropas considerándose que el nuevo orden internacional se caracterizará por el consenso de que la fuerza no ha de utilizarse para resolver disputas y que cuando esto ocurra las cargas y responsabilidades serán compartidas por muchas naciones". ${ }^{14}$ Sin embargo, establece que este nuevo orden internacional no es una pax americana,

\footnotetext{
${ }^{13}$ Citas tomadas del ensayo "Chile y el nuevo orden mundial", coordinado por el profesor Gustavo Lagos Matus.

${ }^{14} \mathrm{Ibid}$
} 
sino que un intento de desalojar la agresión y resistirla si es necesario mediante medidas colectivas y voluntarias de la comunidad internacional.

En contraposición con lo expresado por el Vice-Consejero de Seguridad Gates, pueden destacarse las opiniones de un ex-Ministro de Defensa francés y de un distinguido profesor norteamericano. Jean-Pierre Chevenement, que renunció a sủ cargo de Ministro precisamente por discrepancias con su gobierno por la participación francesa en la Guerra del Golfo, dijo: "El Golfo marcó el advenimiento de un orden internacional dominado por Estados Unidos en el cual la oposición Este-Oeste se sustituye por la oposición Norte-Sur. No se trata de un nuevo orden fundado en el Derecho sino sobre la hegemonía norteamericana reafirmada por la fuerza y que se traduce en un nuevo lenguaje en las relaciones Norte-Sur, en una demostración de fuerza brutal que puede condenar a regiones enteras a la anarquía, pero que está perfectamente admitida por el Norte en tanto tenga por teatro los países del Sur. Un telón de fondo se ha abatido entre los dos polos del mundo como el que antes había entre Este y Oeste; más allá del mismo la reglas son diferentes" ${ }^{15}$ En relación con la situación de los países del Sur su opinión es: "renunciar a defender en dos tercios del planeta la universalidad de los valores que defendemos en nuestra propia casa sería la filosofía del apartheid, un apartheid a escala mundial."

Richard Falk, profesor de Derecho Internacional del Centro de Estudios Internacionales de la Universidad de Princeton, Estados Unidos, se refirió en los siguientes términos al rol de las Naciones Unidas durante la Guerra del Golfo: "Desde la iniciación de la Guerra del Golfo, el rol de las Naciones Unidas ha disminuido a cero. Para todo el mundo se hizo evidente que, para bien o para mal, esta guerra ha sido el resultado de decisiones tomadas en Washington por los líderes políticos norteamericanos actuando por si solos". ${ }^{17}$ En un estudio de las resoluciones del Consejo de Seguridad en relación con la crisis del Golfo opinó: "Las Naciones Unidas se convirtieron en un virtual instrumento de la política exterior norteamericana comprometiendo así su credibilidad en el futuro."18 
A. Toro D. / El espacio en la perspectiva de un nuevo ...

Según su opinión, la situación que se enfrentó en la Guerra del Golfo, fue la consecuencia de la ilusión que los gobiernos y la opinión pública se habían forjado de que existía una diplomacia de las Naciones Unidas, la que en realidad no era propia de ella sino que estaba manejada por Estados Unidos y de que en muchos Estados se había llegado a un estado de pasividad durante la Guerra Fría, entregando la paz y la seguridad del mundo al arbitrio de las dos superpotencias. Para Falk, la continuación de esta pasividad puede producir una nueva y peligrosa misión de la pax americana.

En cuanto a la existencia de un nuevo orden internacional, en la Reunión Cumbre de Londres de las siete potencias más industrializadas, entre las cuales está Estados Unidos, se emitió una declaración conjunta refiriéndose no a un nuevo orden sino que al fortalecimiento del actual, vigorizando el rol de las Naciones Unidas.

El Doctor en Derecho de la Universidad de Navarra, Francisco Balart, ${ }^{19}$ refiriéndose a la forma futura del modus vivendu entre los Estados, analiza cuatro diferentes posibles escenarios o sistemas:

- Sistema monopolar, con una hegemonía norteamericana. "Obviando la espinosa cuestión de si los Estados Unidos de América están a la altura de lo que el peso de la púrpura supone, la desintegración del bloque comunista le abre la posibilidad de ejercer hegemónicamente el liderazgo del sistema internacional. ${ }^{20}$

- Sistema bipolar, "similar al antiguo pero desideologizado, tomando en cuenta que la Federación Rusa mantiene su capacidad nuclear que se estimó en aproximadamente 30.000 ojivas. Así, el autor citado establece que "la Unión Soviética, pese a su innegable derrota ideológica, no está a merced del vencedor" ${ }^{\text {. }} 21$

- Sistema multipolar, cuya característica es la existencia de tres fuertes bloques: uno liderado por Estados Unidos, el otro por Japón y el tercero por Alemania.

Por otra parte se podría mantener lo que se denominó como el sistema pentagonal con Estados Unidos, Rusia, China, Japón y Europa.

- Finalmente, el cuarto sistema o escenario sería uno basado en la dispersión del poder a nivel internacional, donde "el nuevo orden

\footnotetext{
19 "Nuevo Orden Internacional y Estratégico", publicación del Seminario Bases de una Polftica Nacional frente al Nuevo Orden Mundial.

${ }^{20} \mathrm{Ibid}$

${ }^{21}$ Ibid.
} 
internacional no sería ya la competencia por la hegemonía entre un par de superpotencias o entre tres o cinco bloques más o menos autónomos entre sî". "A escala internacional, el poder se fragmentaría concentrándose en múltiples áreas regionales, ninguna de las cuales podría aspirar al control del sistema en su conjunto. ${ }^{\text {222 }}$

Por mucho tiempo, el uso de satélites militares fue compartido por Estados Unidos y la Unión Soviética en forma exclusiva. El derrumbe soviético dejó a los Estados Unidos como la potencia que domina sin contrapeso el espacio ultraterrestre. La ex-Unión Soviética tiene una capacidad espacial que está en decadencia por cuanto carece de los recursos económicos para mantenerla y menos para incrementarla.

También resulta innegable que los países en desarrollo, y aún más los subdesarrollados, no tendrán por mucho tiempo capacidad para jugar un rol en el espacio ultraterrestre y sólo podrán aprovechar, cuando les sea permitido, la capacidad de las naciones ricas llegándose así, al estado de apartheid científico-tecnológico. Sería una tecnocracia internacional. ${ }^{23}$

Tomando en consideración las diferentes opiniones anteriormente expuestas en relación con las interrogantes planteadas al iniciar este último capítulo, sería posible establecer algunas presuntas conclusiones, todas ellas basadas en posibilidades y visiones parciales $\mathrm{y}$, por lo tanto, propias de discusiones por cuanto no son sentencias de verdad absoluta:

a) No existe una clara definición de las características de un nuevo orden político-estratégico internacional ni tampoco de un fortalecimiento del orden internacional existente hasta antes de la Guerra del Golfo.

b) La no existencia de contraposiciones de intereses en la controversia Este-Oeste, ha puesto en evidencia con mayor intensidad las diferencias entre los mundos Norte y Sur.

c) Estados Unidos por si solo, pero con mayor seguridad con el apoyo de los países rectores del Norte, tendrían la posibilidad de vulnerar en el Tercer Mundo, valores que consideran trascendentes en sus propios países si ello conviene a sus intereses.

22 Ibid

${ }^{23}$ Referencia del Sr. Ministro Chevenement. 
A. Toro D. / El espacio en'la perspectiva de un nuevo ...

d) El Consejo de Seguridad de las Naciones Unidas no da plena confianza que la paz y la seguridad del mundo puedan ser mantenidas con justicia, en tanto no se modifique su estructura actual y no esté sujeto a presiones de potencias líderes.

e) El uso del espacio ultraterrestre debería estar abierto a todas las naciones del mundo y al margen del apartheid científico-tecnológico que se produce en la actualidad y que reduce sus posibilidades de participación o que las condiciona.

f) Asimismo, la capacidad político-estratégica de dominio del espacio ultraterrestre por sólo una superpotencia deja a todas las naciones del mundo y, especialmente a las del Tercer Mundo, en una desmedrada situación al poder ser vigiladas, controladas e interferidas cuando los intereses de ella o de otras potencias afines política, militar y económicamente se sientan vulnerados, ya que el control del espacio exterior y las inmensas posibilidades futuras que presenta su uso militar se han constituido en un factor de preponderancia en la definición y materialización del potencial nacional de los Estados. 